\title{
Stabilizing homogeneous black strings in AdS
}

\author{
Adolfo Cisterna, ${ }^{a}$ Carla Henríquez-Báez ${ }^{b}$ and Julio Oliva ${ }^{b}$ \\ ${ }^{a}$ Vicerrectoría Académica, Universidad Central de Chile, \\ Toesca 1783, Santiago, Chile \\ ${ }^{b}$ Departamento de Fúsica, Universidad de Concepción, \\ Casilla 160-C, Concepción, Chile \\ E-mail: adolfo.cisterna@ucentral.cl, carlah.baez@gmail.com, \\ juoliva@udec.cl
}

ABSTRACT: It is known that homogenous black strings in flat spacetime are unstable under long wavelength perturbations. Recently, by including scalar fields that depend on the extended coordinates only, homogenous black strings have been constructed in the presence of a negative cosmological constant, therefore implying that generic perturbations will contain both metric and scalar fluctuations. Here we show that for such generic perturbations homogeneous black strings with a negative cosmological constant are perturbatively stable at linear level, regardless the size of the Schwarzschild-AdS black hole located on the brane.

Keywords: Black Holes, Classical Theories of Gravity, p-branes

ARXIV EPRINT: 1909.05404 


\section{Contents}

1 Introduction 1

2 Homogeneous black strings in AdS 2

3 Linear perturbations $\quad 2$

4 Further remark 4

\section{Introduction}

In General Relativity (GR) in vacuum, one can trivially oxidate black holes to create homogeneous black strings and black $p$-branes in higher dimensions. These objects are an explicit example of the new phenomena that higher-dimensional gravity offers [1]. Indeed, they exhibit a very rich dynamics since they were proved to suffer from the so-called Gregory-Laflamme (GL) instability [2], a long-wavelength perturbative instability triggered by a mode that travels along the extended direction of the horizon, mimicking the spectrum of the Rayleigh-Plateau instability in fluid dynamics [3]. Beyond the validity of the linear regime, there is enough evidence that for long times, the non-linear effects of GR trigger a violation of cosmic censorship, since the black string horizon will pinch-off in a finite amount of time as seen by an asymptotic observer $[4,5]$. Such instability pervades asymptotically flat, black objects in higher dimensions, leading to a similar pinch-off of the rapidly rotating Black Ring horizon [6], as well as of the rapidly rotating Myers-Perry black hole in dimensions $D \geq 6$ [7]. It is known that the inclusion of a negative cosmological constant spoils any direct, homogeneous oxidation of the asymptotically AdS black holes (see e.g. [8]), nevertheless, as recently reported in [9] this obstruction can be circumvented in five dimensions by the presence of a massless scalar $\psi(y)$ such that $d \psi(y)=\lambda d y$, where the integration constant $\lambda$ turns out to be fixed in terms of the bared cosmological constant, and $y$ stands for the coordinate along the extended direction. ${ }^{1}$ This construction can be extended to flat $p$-branes by the introduction of $p$ massless scalars $\psi_{i}\left(y^{i}\right)$ with $i=1, \ldots, p$ such that

$$
d \psi_{1} \wedge \ldots \wedge d \psi_{p} \sim d y^{1} \wedge \ldots \wedge d y^{p}
$$

with $y^{i}$ the Cartesian coordinates of the extended flat directions. The purpose of the present work is to prove the linear, mode stability of these configurations under generic perturbations. These perturbations lead to an instability in the asymptotically flat case, nevertheless, in the presence of a cosmological constant they do not trigger any instability at all.

\footnotetext{
${ }^{1}$ These configurations can be interpreted as Freund-Rubin backgrounds [10] for a fundamental 0-form, with a 1-form field strength $d \psi$ that is proportional to the volume form of the extended direction.
} 


\section{Homogeneous black strings in AdS}

Homogenous black strings and $p$-branes are constructed out from $D=d+p$ dimensional spacetimes of the form

$$
\stackrel{\circ}{g}_{A B} d x^{A} d x^{B}=g_{\mu \nu} d x^{\mu} d x^{\nu}+\delta_{i j} d y^{i} d y^{j}
$$

where the $D$-dimensional metric $\stackrel{\circ}{g}_{A B}$ is the direct product of a $d$-dimensional black hole metric $g_{\mu \nu}$ and a $p$-dimensional flat Riemannian metric $\delta_{i j}$. To circumvent the evident incompatibility of the field equations induced by the presence of a negative cosmological constant, we endow the theory with $p$ minimally coupled scalar fields $\psi_{i}=\psi_{i}\left(y^{i}\right)$, that dress the extended coordinates in such a manner that no incompatibilities arise [9]. Then, the Einstein-Klein-Gordon system

$$
\begin{aligned}
G_{A B}+\Lambda g_{A B} & =\sum_{i}^{p} \frac{\kappa}{2}\left(\partial_{A} \psi_{i} \partial_{B} \psi_{i}-\frac{1}{2} g_{A B}\left(\partial \psi_{i}\right)^{2}\right), \\
\square \psi_{i} & =0,
\end{aligned}
$$

admits the following, homogeneous black string solution

$$
\begin{aligned}
d s^{2} & =\stackrel{\circ}{g}_{A B} d x^{A} d x^{B} \\
& =-f(r) d t^{2}+\frac{1}{f(r)} d r^{2}+r^{2} d \sigma_{d-2}^{2}+\delta_{i j} d y^{i} d y^{j},
\end{aligned}
$$

where $d \sigma_{d-2}$ stands for the line element of a $(d-2)$-dimensional hypersphere and

$$
\begin{aligned}
f(r) & =1-\frac{2 m}{r}-\frac{2 \Lambda}{(d-1)(d+p-2)} r^{2} \\
\lambda^{2} & =-\frac{4 \Lambda}{\kappa(d+p-2)} \\
\psi_{i} & =\lambda y^{i} .
\end{aligned}
$$

Clearly, the bared cosmological constant $\Lambda$ must be negative, and for further simplicity, and without losing generality it will be fixed in such a way that for the corresponding spacetime dimension, $\lambda=1$.

The next section is devoted to prove the stability, under generic linear perturbations of these configurations. For the sake of concreteness we focus on the five-dimensional case, even though we provide some comments about the case in arbitrary dimension in the conclusions.

\section{Linear perturbations}

The five-dimensional AdS black string is given by configuration (2.5) with

$$
\begin{aligned}
f(r) & =-\frac{2 \Lambda r^{2}}{9}-\frac{2 m}{r}+1, \quad \lambda^{2}=-\frac{4 \Lambda}{3 \kappa} \\
\psi & =\lambda y
\end{aligned}
$$


on which we fix $\kappa=2$ and $\Lambda=3 / 2$ such that $\lambda=1$. A generic, spherically symmetric perturbation of this metric will contain both, a fluctuation of the scalar field as well as a fluctuation of the metric, therefore, as for the asymptotically flat black string we introduce the following metric perturbation $g_{A B} \rightarrow \stackrel{\circ}{g}_{A B}+\epsilon h_{A B}$ with

$$
h_{A B}(r)=e^{\Omega t+i k y}\left(\begin{array}{cccc}
h_{1}(r) & h_{2}(r) & 0 & h_{6}(r) \\
h_{2}(r) & h_{3}(r) & 0 & h_{7}(r) \\
0 & 0 & h_{4}(r) \sigma_{2} & 0 \\
h_{6}(r) & h_{7}(r) & 0 & h_{5}(r)
\end{array}\right),
$$

while for the scalar field we consider

$$
\psi \rightarrow \stackrel{\circ}{\psi}+\epsilon \chi=y+\epsilon \Phi(r) e^{\Omega t+i k y} .
$$

Clearly the metric and scalar field fluctuations preserve the spherical symmetry of the background. Here $\epsilon$ is the perturbative parameter. If we are able to find bounded solutions of the linearized system, for positive values of $\Omega$, we would have found an unstable mode, while the absence of such modes will imply the linear stability of our configuration. The linearized Einstein-Klein-Gordon system leads to

$$
\begin{aligned}
& -\frac{1}{2} \stackrel{\circ}{\triangle}_{L} h_{A B}+\frac{1}{2} \stackrel{\circ}{g}_{A B} h^{C D} \stackrel{\circ}{R}_{C D}+\frac{1}{4} \stackrel{\circ}{g}_{A B} \stackrel{\circ}{g}^{C D} \triangle_{\triangle_{L}} h_{C D}+h_{A B}\left(\Lambda-\frac{1}{2} \stackrel{\circ}{R}\right)=T_{A B}^{(1)}, \\
& \stackrel{\circ}{\square}-\stackrel{\circ}{\nabla}_{A} h^{A B} \stackrel{\circ}{\nabla}_{B} \dot{\psi}-h^{A B} \stackrel{\circ}{\nabla}_{A} \stackrel{\circ}{\nabla}_{B} \stackrel{\circ}{\psi}+\frac{1}{2} \stackrel{\circ}{\nabla}_{A} h \stackrel{\circ}{\nabla}^{A} \stackrel{\circ}{\psi}=0,
\end{aligned}
$$

where $\triangle_{L}$ is the Lichnerowicz operator of the background spacetime, and

$$
\begin{aligned}
T_{A B}^{(1)}= & \frac{1}{2}\left(\partial_{A} \dot{\psi} \partial_{B} \chi+\partial_{A} \chi \partial_{B} \stackrel{\circ}{\psi}\right)-\frac{1}{4} \stackrel{\circ}{g}_{A B} \stackrel{\circ}{g}^{C D}\left(\partial_{C} \psi \partial_{D} \chi+\partial_{C} \chi \partial_{D} \stackrel{\circ}{\psi}\right) \\
& -\frac{1}{4} \partial_{C} \dot{\psi} \partial_{D} \stackrel{\circ}{\psi}\left(h_{A B} \stackrel{\circ}{g}^{C D}-\stackrel{\circ}{A}_{A B} h^{C D}\right) .
\end{aligned}
$$

In the presence of matter in GR, it is consistent to impose the following gauge condition

$$
\stackrel{\circ}{\nabla}_{A} h^{A B}=\frac{1}{2} \stackrel{\circ}{\nabla}^{B} h_{C}^{C},
$$

which reduces the equation for the scalar field fluctuation (3.5) to

$$
\stackrel{\circ}{\square} \chi-h^{A B} \stackrel{\circ}{\nabla}_{A} \stackrel{\circ}{\nabla}_{B} \stackrel{\circ}{\psi}=0 .
$$

Even more, the second term vanishes since for the background metric (2.5), the components $\stackrel{\circ}{\Gamma}_{A B}^{y}$ of the Christoffel symbol identically vanish, and we are therefore left with the massless Klein-Gordon equation on the background metric, for the scalar field fluctuation. Introducing the separation (3.4) one is left with the following ODE

$$
\begin{array}{r}
\left(r-r_{+}\right)^{2}\left(r^{2}+r_{+} r+r_{+}^{2}+3\right) r \frac{d^{2} \Phi}{d r^{2}}+ \\
\left(r-r_{+}\right)\left(4 r^{3}-r_{+}^{3}+6 r-3 r_{+}\right)\left(r^{2}+r_{+} r+r_{+}^{2}+3\right) \frac{d \Phi}{d r}-3 r^{2}\left(\left(r-r_{+}\right) k^{2}+3 r \Omega^{2}\right) \Phi=0 .
\end{array}
$$


The regular asymptotic behaviors provided by this equation, near the horizon and infinity yield

$$
\begin{array}{ll}
\Phi(r) \sim\left(r-r_{+}\right)^{\frac{r_{+} \Omega}{r_{+}^{2}+1}}\left(1+\mathcal{O}\left(r-r_{+}\right)\right) & \text {as } r \rightarrow r_{+}, \\
\Phi(r) \sim r^{-\frac{3}{2}-\frac{\sqrt{12 k^{2}+9}}{2}}\left(1+\mathcal{O}\left(r^{-1}\right)\right) & \text { as } r \rightarrow+\infty .
\end{array}
$$

For positive values of $\Omega$, connecting these two asymptotic behaviors is impossible. This is proved as follows: any bounded solution of (3.9) has to vanish both at infinity and at the horizon, therefore there must exist a turning point in the domain of outer communications. At such point $r=r_{*}>r_{+}$one must have

$$
\frac{1}{\Phi\left(r_{*}\right)} \frac{d^{2} \Phi\left(r_{*}\right)}{d r^{2}}=\frac{3 r_{*}\left(\left(r_{*}-r_{+}\right) k^{2}+3 r_{*} \Omega^{2}\right)}{\left(r_{*}-r_{+}\right)^{2}\left(r_{*}^{2}+r_{+} r_{*}+r_{+}^{2}+3\right)},
$$

and since the r.h.s. is strictly positive, no such turning point exists. This standard argument proves the non-existence of exponentially growing modes in time, for generic perturbations of the black string, and therefore its stability!

\section{Further remark}

We have proved that the same perturbation that leads to the GL instability of asymptotically flat black strings, is not able to trigger an instability of the homogeneous asymptotically $A d S_{4} \times R$ black strings of GR in five dimensions, supported by a massless scalar. The presence of the scalar field therefore stabilizes the black string solution. The results presented above can be extended to arbitrary dimension $D=d+p$, and even beyond spherical symmetry. Indeed, using the same gauge condition (3.7), each of the perturbations of the $p$ scalar fields in equation $(2.8), \delta \psi_{(i)}=\chi_{(i)}$, will solve a massless Klein-Gordon equation on the background metric (2.5). Introducing the separation $\chi_{(i)}=e^{\Omega t+i \vec{k} \cdot \vec{y}} Y_{l}\left(\sigma_{d-2}\right) \Phi_{(i)}(r)$, leads to the ODE

$$
r^{2} f^{2} \Phi_{(i)}^{\prime \prime}(r)+r f\left((d-2) f+r f^{\prime}\right) \Phi_{(i)}^{\prime}(r)-\left(\Omega^{2} r^{2}+l(l+d-1) f+\vec{k}^{2} r^{2} f\right) \Phi_{(i)}(r)=0,
$$

with $i=1, \ldots, p$ and where $Y_{l}\left(\sigma_{d-2}\right)$ stands for a spherical harmonics on the $\sigma_{d-2}-$ sphere. As usual due to the spherically symmetric nature of the background, only the $l$ label of the harmonics appears. The relevant asymptotic behaviours are $\Phi_{(i)} \sim\left(r-r_{+}\right)^{\Omega / f^{\prime}\left(r_{+}\right)}$ and $\Phi_{(i)} \sim r^{-\Delta_{+}}$, with $2 \Delta_{+}:=(d-1)+\sqrt{(d-1)^{2}+4 L^{2} \vec{k}^{2}}$ at the horizon and at infinity, respectively and $L$ is the effective AdS radius of the asymptotic region of the black hole on the brane. As before, equation (4.1) does not admit regular solutions for positive $\Omega$ and therefore, the system is stable.

Many questions are now in order. Recently the authors of [11] proved that the black strings constructed out with black holes with negative heat capacity will be perturbatively unstable, proving under certain conditions, the Gubser-Mitra conjecture [12]. It is well known that small black holes in AdS do indeed have negative heat capacity, nevertheless we have explicitly proved the stability of the black strings regardless the size of the 
Schwarzschild-AdS black hole on the transverse section. It would be interesting to explore how the inclusion of the negative cosmological constant, or the massless scalar allows to circumvent the hypotheses presented in [11]. Normalizing the action as

$$
I=\frac{1}{16 \pi G_{D}} \int \sqrt{-g} d^{D} x\left(R+\frac{(D-1)(D-2)}{l^{2}}-\frac{1}{2}(\partial \phi)^{2}\right)
$$

one can compute the mass and entropy of the black strings, leading to

$$
M_{B S}=\frac{(D-3) V\left(S^{D-3}\right) L}{16 \pi G_{D}} r_{+}^{D-3}\left(1+\frac{(D-1)}{(D-2)} \frac{r_{+}^{2}}{l^{2}}\right), \quad S_{B S}=\frac{V\left(S^{D-3}\right) L r_{+}^{D-3}}{4 G_{D}}
$$

in terms of the radius of the horizons $r_{+}$. The background, with zero mass, is globally $\mathrm{AdS}_{4} \times R$ with a non-vanishing scalar. Given the lapse function of the black string it is direct to compute the temperature $4 \pi T=f^{\prime}\left(r_{+}\right)$, and from there the heat capacity $C=d M / d T$. As a function of the radius of the black hole in the transverse section, the heat capacity changes its sign when $r_{+}=r_{+}^{c}=\left(\frac{D-4}{D-1}\right)^{\frac{1}{2}} l$. As usual, small black holes will lead to black strings with negative heat capacity, while local thermal stability is reached when the black hole on the transverse section has a radius above the critical value. Regardless the sign of the heat capacity, we have proved in the previous section the perturbative, mode, stability of our configurations. One may wonder whether a non-linear analysis would lead to an unstable behavior. At least the ground state $A d S_{4} \times R$ obtained by setting $m=0$ in (2.6), and which requires a non-vanishing scalar field, may suffer from the recently discovered non-linear $A d S_{4}$ instability [13] for some type of initial data with arbitrarily small energy, as it is also natural to expect that there might be initial data that will not collapse to black hole as that identified e.g. in [14]-[16]. How the scalar and the extended directions produce an imprint in such non-linear process is something that certainly deserves further exploration.

\section{Acknowledgments}

We thank Christos Charmousis, Gustavo Dotti, Gaston Giribet, Reinaldo Gleiser, Marcela Lagos and Aldo Vera for enlightening comments. A. C work is supported by Fondecyt Grant 11170274 and Proyecto Interno Ucen I+D-2018 CIP 2018020. C. H acknowledges support from Beca CONICYT Doctorado Nacional (2017) 21171394. J. O. work has been funded by Fondecyt Grant 1181047.

Open Access. This article is distributed under the terms of the Creative Commons Attribution License (CC-BY 4.0), which permits any use, distribution and reproduction in any medium, provided the original author(s) and source are credited. 


\section{References}

[1] N.A. Obers, Black holes in higher-dimensional gravity, Lect. Notes Phys. 769 (2009) 211 [arXiv:0802.0519] [INSPIRE].

[2] R. Gregory and R. Laflamme, Black strings and p-branes are unstable, Phys. Rev. Lett. 70 (1993) 2837 [hep-th/9301052] [INSPIRE].

[3] V. Cardoso and O.J.C. Dias, Rayleigh-Plateau and Gregory-Laflamme instabilities of black strings, Phys. Rev. Lett. 96 (2006) 181601 [hep-th/0602017] [INSPIRE].

[4] M.W. Choptuik, L. Lehner, I. Olabarrieta, R. Petryk, F. Pretorius and H. Villegas, Towards the final fate of an unstable black string, Phys. Rev. D 68 (2003) 044001 [gr-qc/0304085] [INSPIRE].

[5] L. Lehner and F. Pretorius, Black strings, low viscosity fluids and violation of cosmic censorship, Phys. Rev. Lett. 105 (2010) 101102 [arXiv:1006.5960] [INSPIRE].

[6] P. Figueras, M. Kunesch and S. Tunyasuvunakool, End point of black ring instabilities and the weak cosmic censorship conjecture, Phys. Rev. Lett. 116 (2016) 071102 [arXiv: 1512.04532] [INSPIRE].

[7] P. Figueras, M. Kunesch, L. Lehner and S. Tunyasuvunakool, End point of the ultraspinning instability and violation of cosmic censorship, Phys. Rev. Lett. 118 (2017) 151103 [arXiv:1702.01755] [INSPIRE].

[8] R.B. Mann, E. Radu and C. Stelea, Black string solutions with negative cosmological constant, JHEP 09 (2006) 073 [hep-th/0604205] [INSPIRE].

[9] A. Cisterna and J. Oliva, Exact black strings and p-branes in general relativity, Class. Quant. Grav. 35 (2018) 035012 [arXiv:1708.02916] [INSPIRE].

[10] P.G.O. Freund and M.A. Rubin, Dynamics of dimensional reduction, Phys. Lett. B 97 (1980) 233 [INSPIRE].

[11] S. Hollands and R.M. Wald, Stability of black holes and black branes, Commun. Math. Phys. 321 (2013) 629 [arXiv:1201.0463] [INSPIRE].

[12] S.S. Gubser and I. Mitra, Instability of charged black holes in anti-de Sitter space, Clay Math. Proc. 1 (2002) 221 [hep-th/0009126] [INSPIRE].

[13] P. Bizon and A. Rostworowski, On weakly turbulent instability of anti-de Sitter space, Phys. Rev. Lett. 107 (2011) 031102 [arXiv:1104.3702] [INSPIRE].

[14] V. Balasubramanian, A. Buchel, S.R. Green, L. Lehner and S.L. Liebling, Holographic thermalization, stability of anti-de Sitter space and the Fermi-Pasta-Ulam paradox, Phys. Rev. Lett. 113 (2014) 071601 [arXiv:1403.6471] [INSPIRE].

[15] A. Buchel, S.R. Green, L. Lehner and S.L. Liebling, Conserved quantities and dual turbulent cascades in anti-de Sitter spacetime, Phys. Rev. D 91 (2015) 064026 [arXiv:1412.4761] [INSPIRE].

[16] A. Biasi, B. Craps and O. Evnin, Energy returns in global $A d S_{4}$, Phys. Rev. D 100 (2019) 024008 [arXiv:1810.04753] [INSPIRE]. 